\title{
Manifest für eine Post-Kritische Pädagogik
}

Naomi Hodgson, Joris Vlieghe und Piotr Zamojski

Übersetzung ${ }^{1}$ aus dem Englischen von Sabrina Carbone, Anna Lena Sahliger und Martin Bittner, unter Mitarbeit von Sascha Michaelis und Anni Heinrichsen.

Anmerkung der Übersetzer:innen: Wir haben in unserer Übersetzung versucht, die gegebenen komplexen, verschachtelten Satzstrukturen beizubehalten und in der Wortwahl die Radikalität/Strenge abzubilden, die ein Manifest unseres Erachtens mit sich bringt. Gleichsam entsteht durch die Übersetzung neuer Gesprächsbedarf über bestehende Konzepte, verwendete Begriffe in den jeweiligen Sprachräumen und Disziplinen. Eine gemeinsame Sprache auch in der Übersetzung zu finden, bleibt sodann eine spannende Herausforderung im Wissensaustausch (der gerade bei Übersetzungen immer schon im Vordergrund steht).

Grundsätze $\mathrm{zu}$ formulieren, scheint zumindest in der Philosophie der Erziehung auf eine Form normativer, konzeptioneller Analyse zurückzuführen, die mit englischsprachigen Strömungen analytischer Philosophie assoziiert ist. Jedoch bringen die poststrukturalistische und postmoderne Philosophie - zumindest soweit sie in die Bildungstheorie und auch in alltägliche Überlegungen im Generellen aufgenommen wurden - häufig einen Relativismus mit sich, welcher, während er potentiell inklusiv und mit Sicherheit heutzutage grundlegend für die Möglichkeit der individuellen Wahl ist, sich dennoch in der Verteidigung von Grundsätzen als schwierig erweist. Indem

1 Wir danken Jürgen Budde, Anke Wischmann und Laura Cunniff für hilfreiche Nachfragen und Vorschläge an verschiedenen Stellen der Übersetzung. 
Grundsätze in Form eines Manifests formuliert werden, riskieren wir Vorwürfe der Verallgemeinerung (der Universalisierung), ausgenommen der Normativität. Allerdings ist es vielleicht an der Zeit, die Annahme zu hinterfragen, dass diese naturgemäß und immer negativ sind. Im Folgenden stellen wir Grundsätze vor, die gegründet sind auf der Überzeugung der Möglichkeit der Veränderung, wie sie in der kritischen Theorie und Pädagogik zu finden ist, allerdings mit einer affirmativen Haltung: eine post-kritische Ausrichtung von Bildung, die unter aktuellen Bedingungen an Einfluss gewinnen wird und dies wiederum ist begründet in einer Hoffnung für das, was noch kommen wird.

Der erste Grundsatz, welcher hier aufgeführt wird, ist einfach der, dass es Grundsätze zu verteidigen gibt. Dies verpflichtet uns an sich zu nichts Weiterem, wie es sich ausdrückt in, z.B., dass wir $\mathrm{x}$ tun sollten. Das ist nicht Normativität im Sinne der Definition eines Ideals eines gegenwärtigen oder zukünftigen Zustands, an dem die gegenwärtigen Praktiken beurteilt werden sollen. Folglich könnte man diesen Grundsatz als Verteidigung einer Verschiebung von einer prozeduralen Normativität zu einer prinzipientreuen Normativität charakterisieren.

In der Bildungstheorie sind poststrukturalistische und postmoderne Überlegungen im Kontext von Identitätspolitiken aufgegriffen worden, und sind befasst mit Andersartigkeit, Alterität und Mitspracherecht. Respekt für den Anderen und für Differenz erfordern, dass Pädagog*innen akzeptieren, dass wir über den Anderen niemals alles wissen können. Jegliche Bemühung, dies zu erreichen, konstituiert, wenn man so will, "Gewalt« gegen den Anderen. Dementsprechend ist die Möglichkeit $\mathrm{zu}$ handeln und $\mathrm{zu}$ sprechen ausgeschlossen; ein politisches aber auch ein pädagogisches Problem, möglicherweise zusammengefasst in der oft gehörten (wenn auch gemurmelten) Phrase »ich weiß, dass man das so nicht mehr sagen darf, aber..., « und dem Beklagen der sogenannten political correctness. Die Einsicht, dass wir den Anderen nie vollständig verstehen können - Einzelperson oder Kultur -, sollte nicht zur Folge haben, dass wir nicht mehr sprechen können. Solch ein Verständnis von »Respekt « übersieht, dass Verstehen und Respekt fortwährende Herausforderungen und Hoffnungen sind. Hier 
gehen wir von der Annahme aus, dass wir - gemeinsam - sprechen und handeln können und entsprechend vollzieht sich eine Verschiebung der hermeneutischen Pädagogik, die die kritische Pädagogik mit sich bringt, um - einen zweiten Grundsatz - die pädagogische Hermeneutik zu verteidigen. Es ist eben diese Herausforderung des Zusammenlebens in einer gemeinsamen Welt, die jene Hoffnung hervorbringt, Bildung weiterhin als eine erstrebenswerte Aktivität erscheinen zu lassen. Hermeneutik ist kein (unlösbares) Problem, sondern vielmehr etwas, das Pädagog*innen erschaffen müssen. Wir sollten nicht auf der Basis einer apriorischen Annahme über die (Un)Möglickeit eines wirklichen wechselseitigen Verständnisses und Respekts sprechen und handeln, sondern stattdessen zeigen, dass es trotz vieler Differenzen, welche uns voneinander trennen, einen Raum der Gemeinsamkeit gibt, der sich ausschließlich aus der Erfahrung (a posteriori) ergibt (vgl. Arendt, Badiou, Cavell).

Dieser existierende Raum der Gemeinsamkeit wird in vielen erziehungswissenschaftlichen Forschungen, Strategien und Praxen meist übersehen, $\mathrm{zu}$ Gunsten eines Fokus auf soziale (Un)Gerechtigkeit und Exklusion basierend auf Annahmen von Ungleichheit. Das Ethos der kritischen Pädagogik wirkt bis heute fort in der Überzeugung, Gleichberechtigung herzustellen, nicht durch Emanzipation, sondern vielmehr durch die Stärkung (Empowerment) Einzelner und von Gemeinschaften. Allerdings wäre es hoffnungslos - wenn nicht sogar zynisch -, die offensichtliche Unausweichlichkeit der neoliberalen Rationalität darzulegen. Jedoch gibt es diese Notwendigkeit in der gegebenen Ordnung der Dinge nicht, und deshalb gibt es, so unüberwindbar wie die gegenwärtige Ordnung auch erscheint, Hoffnung. Der dritte Grundsatz, der auf der Annahme der Gleichheit (vgl. Rancière) und der Möglichkeit der Transformation des Einzelnen und des Kollektivs beruht, bedeutet also eine Verschiebung von der kritischen Pädagogik zur post-kritischen Pädagogik.

Dies ist keineswegs eine anti-kritische Position. Dank des enormen und äußerst mächtigen kritischen Apparats, der sich im Laufe des 20. Jahrhunderts entwickelt hat, sind wir uns der wesentlichen Merkmale des Status Quo bewusst, in den wir eingebunden sind. Aber im Ge- 
gensatz zur inhärenten Kritik gesellschaftlicher Institutionen, die auf deren Dysfunktionalität fokussiert, oder zur utopischen Kritik, die von einer transzendentalen Position angetrieben wird und zu einem ewigen Aufschub des gewünschten Wandels führt, glauben wir, dass es an der Zeit ist, unsere Bemühungen auf Versuche zu konzentrieren, die unterdrückten Anteile unserer Erfahrung zurückzufordern; wir sehen die Aufgabe einer post-kritischen Pädagogik nicht darin, zu entlarven, sondern zu schützen und zu sorgen (vgl. Latour, Haraway). Diese Sorge und der Schutz manifestieren sich in Form einer erneuten Frage, was Bildung, Erziehung, Unterrichten, Lernen, Denken und Üben sind. Diese Rückforderung bedeutet nicht länger eine kritische Beziehung die offenbart, was wirklich vor sich geht - , noch eine instrumentelle Beziehung - die zeigt, was Pädagog*innen tun sollten -, sondern eröffnen einen Denkraum, der es ermöglicht, dass Praxis von Neuem geschieht. Das bedeutet, unsere Beziehung zu unseren Worten (wieder-)herzustellen, sie zu hinterfragen und den entwerteten Aspekten unserer Lebensweisen philosophische Aufmerksamkeit zu schenken und damit, im Einklang mit einer grundsätzlichen Normativität, diese Ereignisse als Selbstzweck (autotelisch) zu verteidigen, nicht funktionalisiert, sondern einfach weil sie es wert sind, geachtet zu werden.

Bildung basiert in einem sehr praktischen Sinne auf Hoffnung. In der »traditionellen « kritischen Pädagogik jedoch beruht diese Hoffnung auf Emanzipation gegenüber dem existenten Regime der Ungleichheit, die durch diese drei spezifischen Weisen überwunden werden sollen:

1) Es wird eine Art hermeneutischer Pädagogik bemüht: Die Pädagog*in nimmt von den Anderen an, dass ihnen die Fähigkeit fehle, zu verstehen, dass sie in ihrer Sichtweise der Welt verhaftet sind. Die Pädagog*in positioniert sich selbst als außerhalb solcher Strukturen, muss jedoch die Gegenwart kritisieren und die Unaufgeklärten freisetzen (vgl. Platons Höhlengleichnis).

2) In Wirklichkeit kommt es darauf an, die eigene überlegene Position zu bekräftigen, und führt damit zur Wiedereinsetzung eines Regimes der Ungleichheit. Es gibt keinen erkennbaren Bruch zum Status Quo. 
3) Darüber hinaus ist die externe Sichtweise der kritischen Päd-

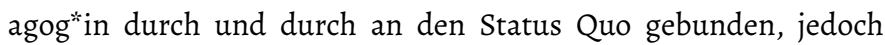
in einer negativen Weise: Die Kritik ist getrieben von der Leidenschaft zum Hass. Auf diese Weise beharrt sie oder er verstohlen auf dem, was ist und was immer sein soll. Richtende und dialektische Ansätze geben diese negative Haltung wieder.

Somit nimmt die Pädagog*in die Rolle der"jenigen ein, die erforderlich ist, um den Schleier zu lüften; allerdings lüften sie den Schleier abhängig vom als unveränderlich angesehenen Status Quo und von ihrem/seinem externen Standpunkt aus. Um die Rolle der Pädagog*in etwas positiver darzustellen, als eine Initiation der neuen Generationen in eine gemeinsame Welt, eröffnen wir die Idee einer post-kritischen Pädagogik, die die Liebe zur Welt einfordert. Dies ist keine Akzeptanz der Dinge, wie sie sind, sondern eine Bejahung der Wertigkeit dessen, was wir in der Gegenwart tun und der Dinge, von denen wir annehmen, dass sie es wert sind, weitergegeben zu werden. Jedoch nicht so, wie sie sind: Die pädagogische Hoffnung richtet sich an die Möglichkeit der Erneuerung unserer gemeinsamen Welt. Wenn wir die Welt, unsere Welt, wirklich lieben, müssen wir gewillt sein, die Welt in die Hände der nächsten Generation zu geben, mit der Annahme, dass sie - die Neuankömmlinge - die Welt nach ihren eigenen Bedingungen fortführen. Somit bringt der vierte Grundsatz eine Verschiebung vom grausamen Optimismus (vgl. Berlant) zur Hoffnung in der Gegenwart mit sich. Zynismus und Pessimismus erkennen nicht an, wie die Dinge sind, sondern sie vermeiden sie (vgl. Cavell, Emerson).

In gegenwärtigen Formulierungen ist die Sorge für die Welt eingerahmt in Begriffe einer Bildung für die Bürgerschaft, Bildung für soziale Gerechtigkeit, Bildung für Nachhaltigkeit etc. hinsichtlich einer bestimmten Vorstellung globaler Bürgerschaft und einer unternehmerischen Form eines interkulturellen Dialogs. Obwohl möglicherweise gestützt von einer progressiven, kritischen Pädagogik, ist die Sorge solcher Ansätze für die Verantwortlichkeit für die Welt letztendlich außerhalb der Pädagogik. So traditionell oder konservativ es vielleicht klingen mag, wünschen wir uns, die Bildung um der Bildung willen zu verteidi- 
24 Naomi Hodgson, Joris Vlieghe und Piotr Zamojski

gen: Bildung als die Erforschung oder die Einführung in einen Gegenstandsbereich für ihren intrinsischen, eher erzieherischen als instrumentellen Wert, so dass dies von der neuen Generation neu aufgegriffen werden kann. Gegenwärtig ist die (zukünftige) Welt bereits eingenommen von diesem »Bildung für ..." und wird instrumentalisiert für (unsere) andere(n) Zwecke. Schlussfolgernd führt uns der fünfte Grundsatz von der Bildung für Bürgerschaft zur Liebe für die Welt. Es ist an der Zeit, das Gute in der Welt, welches es wert ist, es zu bewahren, anzuerkennen und zu befürworten. Es ist an der Zeit, die Welt zu entlarven, um durch hoffnungsvolle Anerkennung der Welt Erfolg zu haben. Es ist an der Zeit, das, was gut ist in der Welt - das, was bedroht ist und das, was wir uns zu erhalten wünschen -, in den Fokus unserer gegenwärtigen Aufmerksamkeit zu stellen und einen konzeptuellen Raum zu schaffen, in dem wir die Verantwortung für sie übernehmen können, im Angesicht und trotz aller Unterdrückung und stummen Melancholie. 